Journal of Computer Science 8 (4): 494-498, 2012

ISSN 1549-3636

(C) 2012 Science Publications

\title{
Low-Loss Hand-Made Demultiplexer using Transparent Color Filter in WDM-POF Network for Short-Haul Communication System
}

\author{
Mohd Syuhaimi Ab. Rahman, L.S. Supian, \\ Hadi Guna, Mohd Hazwan Harun and Kasmiran Jumari \\ Department of Electrical, Electronics and Systems Engineering, \\ Faculty of Engineering and Built Environment, \\ University Kebangsaan 43600 UKM Bangi, Selangor, Malaysia
}

\begin{abstract}
Problem statement: Polymer optical fiber has advantages compared to other alternatives communication media such as copper, coax cable and glass fiber. POF has bigger bandwidth compared to wireless communication network and POF is also free from electromagnetic disruption. Since POF is suitable for communication system up to distance of $100 \mathrm{~m}$ with data speed of 400 Mbps using polymer step-index type, therefore, POF is effective for short haul data transmission. Application of POF includes the Ethernet network at home or usually known as Fiber To The Home (FTTH) and in also used in automotive system. However, since the demand of high capacity of the transmission system, wavelength division multiplexing technique or WDM is applied in this study. Approach: Several signals with different wavelengths are multiplexed and carried over a single fiber and demultiplexed at the end of the communication system to obtain the original signal to be directed to the receiver. The advantage of the device developed in this study is that it is easy to construct and inexpensive. Thin-film is used as filter and three main color wavelength used are red, blue and green thin-films. The red thin-film will absorb any other wavelength other than $\lambda=650 \mathrm{~nm}$, blue thin-film will absorb any other wavelength other than $\lambda=475 \mathrm{~nm}$ while the green thin-film will absorb any other wavelength that is not around $\lambda=510 \mathrm{~nm}$. Characterization is done to test the signal loss through each of the thin-film. Low-loss filter for the wavelength of the desired signal is analyzed to get the best thin-film that gives optimum transmission while blocking other undesired wavelengths. Insertion loss and power output are measured to determine the thin-film to develop the demultiplexer that work effectively. In this study, the distance of the optical fiber is not taken into account, however, the length of the optical fiber is set to be $5 \mathrm{~m}$ each. Results: Although there exists attenuation of signal transmission along the fiber and at the transparent colored thin-film, the demultiplexer works efficiently for short haul communication system with small loss of power output and small increase of insertion loss for the desired wavelength transmitted. Conclusion: Analysis shows that the device works efficiently for short distance communication although the results show insignificant signal loss along the transmission.
\end{abstract}

Key words: Inexpensive, FTTH, high transmission, thin-film, wavelengths filter

\section{INTRODUCTION}

The demand of the utilization of optical fiber is increasing due to the ability of the optical fiber to transmit signal or data with higher speed compared to copper or coax cable. Common advantages of polymer optical fiber (POF) over copper cables are the nonexistent susceptibility to any kind of electromagnetic interference and it has lower weight. More than that, POF has smaller radius of curvature and is more flexible (Haupt et al., 2006). By using multimode type of optical fiber, signal transmission range is between 10Mbps to 10Gbps (Horak, 2007). Multimode fiber can conduct several light rays and can operate free of disruption. Many different wavelengths can be sent simultaneously in a fiber in range of spectrum between $1300 \mathrm{~nm}$ to $1600 \mathrm{~nm}$. This technology of combining a number of wavelengths through a fiber is known as wavelength division multiplexing or WDM (Keiser, 2008). Several

Corresponding Author: Supian, L.S., Department of Electrical, Electronics and Systems Engineering,

Faculty of Engineering and Built Environment University Kebangsaan 43600 UKM Bangi,

Selangor, Malaysia Tel: +603-89216837 
independent signals that have been optically formatted information streams where each is transmitted at a different wavelengths are combined using an optical multiplexer and sent over a same fiber. Each of the signals or data stream maintains its individual information carried within although each signals could be at a different rate. Even after all the signals have been multiplexed into a fiber, each individual signal operates at its unique wavelength (Keiser, 2008). All the different wavelengths that are transmitted over a fiber have to be separated at the receiver to obtain the original signal and direct each of the signal to its particular end-user. This method can be realized by using demultiplexer. There are existing demultiplexer systems available in the market, however, one disadvantage is the high cost of implementation that limits the optical fibers to be fully utilized by the users or the industry. The study is based on the WDM-POF technique where several wavelengths are combined and separated using optical splitter and color filter. In this system, there are several important components such as multiplexer, optical splitter, demultiplexer and colored thin-films. In this study, the demultiplexer sends three different signals with different wavelengths specified by $\operatorname{Red}(\lambda=650 \mathrm{~nm})$, Blue $(\lambda=475 \mathrm{~nm})$ and Green $(\lambda$ $=510 \mathrm{~nm}$ ) LED sources. In theory, each wavelength carried different data and multiplexed over a same fiber and separated into its original signal using demultiplexer at the end of the system. Thus, colored thin-films are used in this study to realize the technique discussed. Moreover, the hand-made demultiplexer using the thin-film consumed very low budget yet effective for data transmission over short distance with high speed. Early stage of the study focuses on selection of thin-films for the development of demultiplexer. Three main wavelengths used are as mentioned above. The three main signals injected by LED sources that are Red, Blue and Green are combined over a single fiber using $1 \times \mathrm{N}$ splitter and the signals combined will then be separated or filtered at each end of the splitter. $1 \times 3$ splitter is used in this study, therefore at the end of the splitter, each of the colored yet transparent thin-films are attached to the fibers that functions as wavelength filter (Ab-Rahman et al., 2009a). Red, blue and green thin-films are selected accordingly and tested as to choose the best thin-film to develop effective demultiplexer. In theory, when the combined signals went through the handmade optical splitter and channel out to the three output, for instance, the Red thin-film will only transmit wavelength of the signal around $\lambda=650 \mathrm{~nm}$ and block or absorb other signal streams of not the same wavelength. In other word, the thin-film filters out the other unwanted signal from being sent to the particular receiver. Same situation happens at the Blue and Green thin-film attached at the end of the splitter. Therefore, the selection of the best thin-film is very crucial in the development of the demultiplexer. Experimental tests for insertion loss $(\mathrm{dBm})$ and power output $(\mathrm{dBm}, \mu \mathrm{W})$ are done and characterization analysis is done in selecting the thin-film that reveals optimum results. Low-loss of signal transmission for the similar wavelength as the thin-film is proven for several thinfilms. These thin-films are effective in filtering out any other unwanted signals from being transmitted over the fiber to the end-receiver. There are a lot of applications using the optical fibers such as internet network at home, in automobile industry and it is very effective in data transmission in short-haul communication network.

Specification of design: The hand-made thin-film based demultiplexer has three wavelength filters specified by Red $(\lambda=650 \mathrm{~nm})$, Blue $(\lambda=475 \mathrm{~nm})$ and Green $(\lambda=510 \mathrm{~nm})$. The thin-films are selected based on low-loss signal transmission for the wavelength specified. The filter is designed to eliminate any unwanted signal and select the wavelength of the system as desired. Experimental tests are conducted to evaluate the effectiveness of the thin-films chosen as filters. Each of the colored thin-film is attached to the end of the three fibers with certain length that are attached with single-position plug assembly or socket as shown in the Fig. 1. The thin-films are glued using epoxy-resin is then secured by inserting single-position bulkhead receptacle as shown in Fig. 2. The bulkhead receptacle also functions as a connector where the other end of the bulkhead will be connected to optical fiber that lead to the receiver.

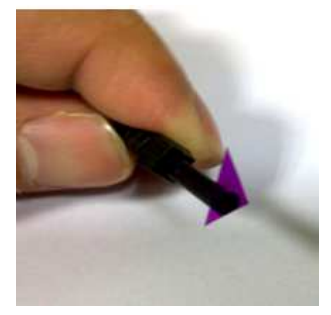

Fig. 1: single-position plug assembly

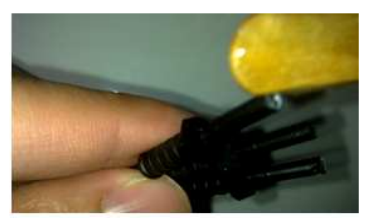

Fig.2: single-position bulkhead receptacle 


\section{MATERIALS AND METHODS}

The samples of the transparent colored thin-film filter are chosen at first stage by analysis of the 'Spectral Energy Distribution' curves provided with each colored thin-film. The curves show the peak of wavelength transmission through the films. Samples are selected based on the optimum transmission of the desired wavelength. The desired wavelength that shows the high peak of transmission and lowest base of other wavelengths is preferred and the sample colored thinfilm is selected for further analysis. The colored thinfilms are made up of two types of plastics which 65\% of its component is made from polycarbonate plastic and the rest is made from polyester dye Roscolux, 1998. Epoxy-resin mixture is used as glue to attach the small pieces of the thin-film samples to the end of the fibers and plug assembly due to its strong adhesive. The rough surface of the polymer optical fibers are refined using sand papers so that low-loss signal transmission can be obtained (Ab-Rahman et al., 2009b).

Approach and method: The first step of the development of the transparent thin-film based demultiplexer is the analysis of the energy spectrum curves provided with each of the colored thin-films. Films that give high peak of desired wavelength transmission are selected before further analysis. Three ranges of desired wavelengths in this study are the Red, Blue and Green. Several samples of each colored thinfilms for red, blue and green are selected based on energy spectrum range. POF cables with desired lengths are connected to the plug assembly or socket. The rough edge surface of the POF is refined using sand papers. Epoxy-resin is used to attach small cut-up thinfilms to the end of the plug assembly and fibers while later on the attachments is left to dry as shown in Fig. 3. After the attachment is completely dried, bulkhead receptacle is inserted with the thin-film attached to optical fiber as in Fig. 4. The other end of the bulkhead receptacle is connected to a socket inserted with short length optical fiber. The short length optical fiber is then connected to the power meter for measurement of insertion loss and power output. Three LED sources are injected through the optical fibers as the input to evaluate the performance of each of the thin-film. Readings are taken for insertion loss $(\mathrm{dBm})$ and power output $(\mathrm{dBm}, \mu \mathrm{W})$. Characterization analysis is done for each of the thin-films that gives optimum transmission for the desired wavelength with minimum loss. In this study, low-loss desired wavelength transmission is evaluated through the small loss of the insertion loss parameter and high power output.

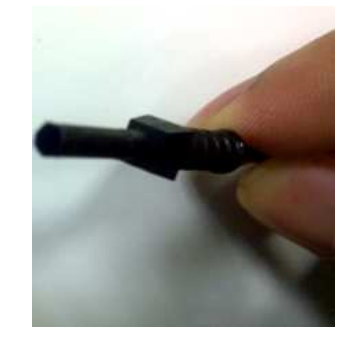

Fig. 3: plug assembly and fibers later attachment

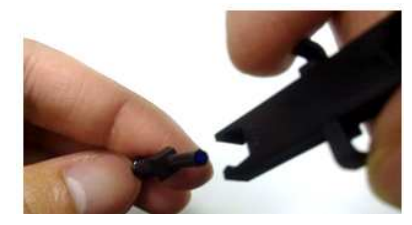

Fig. 4: dried attachment

Characterization: One sample thin-film is chosen for each color of red, blue and green. Characterization test is done for insertion loss and power output. Optical power meter is used to measure the optical power from POFs connected to the thin-films. Before the connection to the thin-film is made, it is obtained that $0.02 \mathrm{~mW}$ of zero error displayed on the meter. The short length POFs cable is then connected to the bulkhead receptacle that connected to the thin film at the other end. For the first stage, only Red LED source is injected through the optical fibers. Analysis is done to observe the high transmission of wavelength around $650 \mathrm{~nm}$ passing through the red thin-film with small loss transmission. Insertion loss value and power output readings are taken and analyzed by plotting the values against the length of the optical fibers. Through this experiment, samples that give low insertion loss and have high transmission percentage will be chosen to develop the demultiplexer that gives optimum results. This study focused on the efficiency of the thin-film to transmit the signal injected with desired wavelength with only small loss. Then, Blue LED source is injected through the optical fiber to obtain the efficiency of the blue thin-film. Same is done to evaluate the efficiency of the green thin-film.

\section{RESULTS}

As shown in Fig. 5, the decrease of power output $(\mu \mathrm{W})$ for Red, Green and Blue LED sources can be seen. It is observed that the transmission for red LED is high since only $4.7 \%$ loss of power output is measured. As shown in Fig. 6, insertion loss (dBm) for red, green and blue LEDs can be seen. 


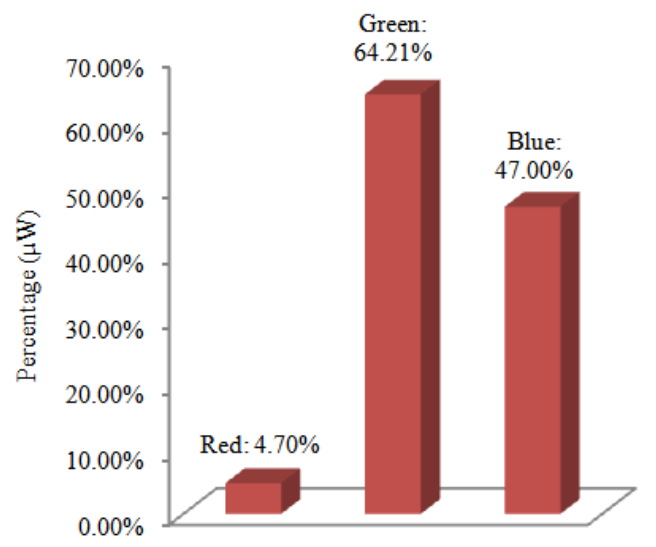

Fig. 5: Decrease of Power Output $(\mu \mathrm{W})$ for Red, Green and Blue LED source injected through the fibers and Red thin-film

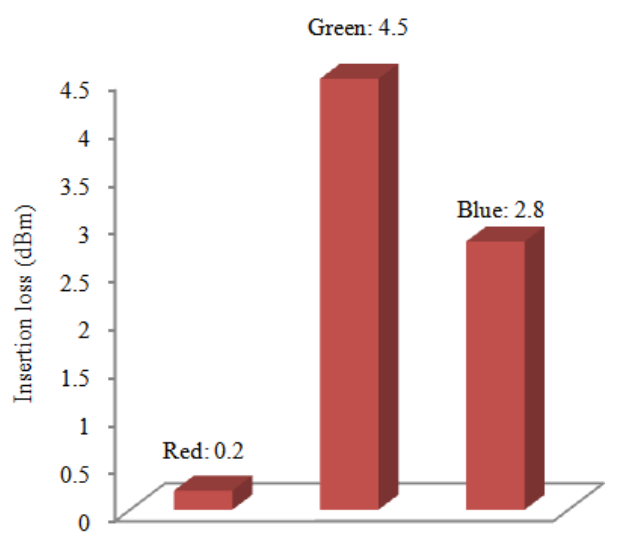

Fig. 6: Increase of Insertion Loss (dBm) for Red, Green and Blue LED source injected through the fibers and Red thin-film.

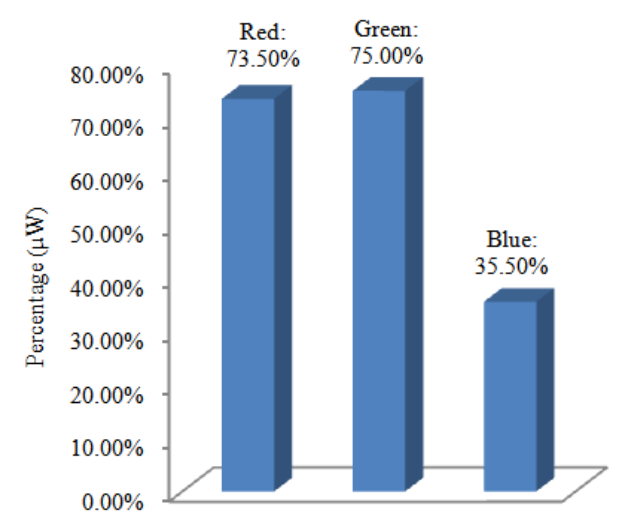

Fig. 7: Decrease of Power Output $(\mu \mathrm{W})$ for Red, Green and Blue LED source injected through the fibers and Blue thin-film

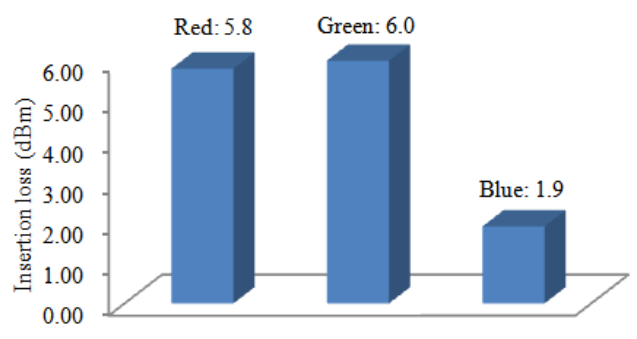

Fig. 8: Increase of Insertion Loss (dBm) for Red, Green and Blue LED source injected through the fibers and Blue thin-film

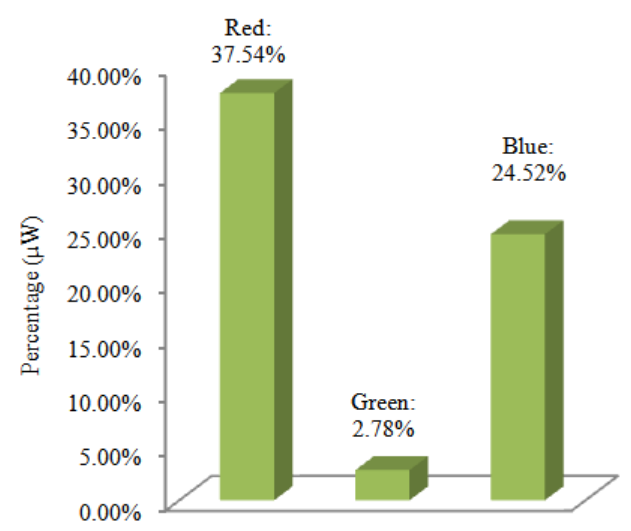

Fig. 9: Decrease of Power Output $(\mu \mathrm{W})$ for Red, Green and Blue LED source injected through the fibers and Green thin-film

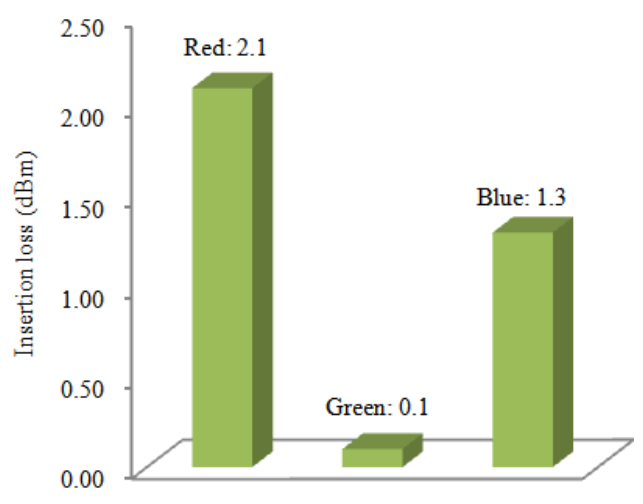

Fig. 10: Increase of Insertion Loss $(\mathrm{dBm})$ for Red, Green and Blue LED source injected through the fibers and Green thin-film

Figure 7 shows the small loss observed for the power output when the blue LED source injected through the blue thin-film. Small increase of insertion loss $(\mathrm{dBm})$ can be observed for blue LED source injected through the blue thin-film as shown in Fig. 8. Similar situation 
is also observed for green LED source injected through three thin films. Small loss of the power output observed for the green LED source as shown in Fig. 9. compared to the red and blue LED sources. Whereas Fig. 10 shows the small loss of insertion loss found for the green LED source transmission through the green thin-film.

\section{DISCUSSION}

Power output loss for green and blue LED source is high since the red thin-film blocks other unwanted wavelength range from being transmitted over. The red thin-film again proves to be efficient since only small insertion loss is observed compared to the insertion loss for green and blue LEDs. High decrease of power output obtained when the red and green LED source went through the blue thin-film. This is due to the different range of wavelength where the blue thin-film blocks undesired wavelengths from being transmitted through the thin-film. Factor that could contribute to the insertion loss and decrease of power output is due to the epoxy-resin utilization on the surface of the optical fibers to attach the thin-films onto the fibers and plug assembly. The glue that spread on the surface of the optical fibers lead to the attenuation of the signal transmitted.

\section{CONCLUSION}

In conclusion, the demultiplexer developed using transparent colored thin-film is effective to send data for short distance communication. The fabrication and characterization analysis has been done successfully to develop demultiplexer with optimum performance for short communication purpose. Red wavelength source $(\lambda=650 \mathrm{~nm})$ is injected through the optical fiber attached with the red, blue and green thin-film that acts as filter for the purpose of characterization test for the signal transmission to see the loss revealed when the source injected. The analysis shows that the signal transmission decreases at small percentage when the desired range of wavelength of signal went through the red thin-film. Similar performance is observed when the Blue $(\lambda=475 \mathrm{~nm})$ and Green $(\lambda=510 \mathrm{~nm})$ LED sources are injected through the fibers and the thinfilms. The loss of the signal transmission of the blue source injected through the blue thin-film is very low which gives optimum performance of signal transmission. Same performance is seen when the green source injected through the fibers and demultiplexer. The demultiplexer developed using thin-films selected based on the characterization test in this study able to give optimum performance for the short haul communication system. Although the maximum distance of the transmission using the device developed has not yet determined, however, in the future, deeper study and research will be done to improve the performance. This device is not only effective for data transmission for short haul communication, but it is also easy to develop and inexpensive. Based on the analysis and characterization of the demultiplexer, the efficiency of the thin-film achieved $70 \%$ efficiency. Although the characterization shows some signal loss along the transmission, the measurement and test done on the data and video transmission depicts optimum performance based on the spectrum analyzer tool.

\section{ACKNOWLEDGMENT}

The researchers would like to thank Spartech Lab group, University Kebangsaan Malaysia for the support of this project.

\section{REFERENCES}

Ab-Rahman, M.S., H. Guna and M.H. Harun, 2009a. Cost-effective $1 \times 12$ POF-based optical splitters as an alternative optical transmission media for multipurpose application. Int. J. Comput. Sci. Netw. Secu., 9: 72-78.

Ab-Rahman, M.S., H. Guna, M.H. Harun, S.D. Zan and K. Jumari, 2009b. Cost-effective fabrication of self-made $1 \times 12$ polymer optical fiber-based optical splitters for automotive application. Am. J. Eng. Applied Sci., 2: 252-259. DOI: 10.3844/ajeassp.2009.252.259

Haupt, M., C. Reinboth and U.H.P. Fischer, 2006. Realization of an economical polymer optical fiber demultiplexer. Proceedings of the International Students and Young Scientists Workshop, Photonics and Microsystems, Jun. 30-Jul. 2, IEEE Xplore Press, Wroclaw, pp: 19-23. DOI: 10.1109/STYSW.2006.343661

Horak, R., 2007. Telecommunications and Data Communications Handbook. 2nd Edn., John Wiley and Sons, Hoboken, N.J., ISBN: 0470041412, pp: 791.

Keiser, G., 2008. Optical Fiber Communications. 1st Edn., Tata McGraw-Hill Education, ISBN: 0070648107, pp: 580. 